\title{
Oral microbial profile variation during canine ligature-induced peri-implantitis development
}

Shichong Qiao ${ }^{1+}$, Dongle Wu ${ }^{1 \dagger}$, Mengge Wang ${ }^{2}$, Shujiao Qian ${ }^{1}$, Yu Zhu', Junyu Shi', Yongjun Wei ${ }^{2 *}$ (iD and Hongchang Lai ${ }^{1 *}$

\begin{abstract}
Background: Dental implants have become well-established in oral rehabilitation for fully or partially edentulous patients. However, peri-implantitis often leads to the failure of dental implants. The aim of this study was to understand the core microbiome associated with peri-implantitis and evaluate potential peri-implantitis pathogens based on canine periimplantitis model.

Results: In this study, three beagle dogs were used to build peri-implantitis models with ligature-induced strategy. The periimplant sulcular fluids were collected at four different phases based on disease severity during the peri-implantitis development. Microbial compositions during peri-implantitis development were monitored and evaluated. The microbes were presented with operational taxonomic unit (OTU) classified at 97\% identity of the high-throughput 165 rRNA gene fragments. Microbial diversity and richness varied during peri-implantitis. At the phylum-level, Firmicutes decreased and Bacteroides increased during peri-implantitis development. At the genus-level, Peptostreptococcus decreased and Porphyromonas increased, suggesting peri-implantitis pathogens might be assigned to these two genera. Further specieslevel and co-occurrence network analyses identified several potential keystone species during peri-implantitis development, and some OTUs were potential peri-implantitis pathogens.
\end{abstract}

Conclusion: In summary, canine peri-implantitis models help to identify several potential keystone peri-implantitis associated species. The canine model can give insight into human peri-implantitis associated microbiota.

Keywords: Microbiota, Ligature-induced, Canine peri-implantitis, Microbial variation, Keystone taxonomy

\section{Background}

With the development of oral technologies, dental implants are a highly successful and predictable treatment for replacing missing teeth, which can rehabilitate oral

\footnotetext{
* Correspondence: yongjunwei@zzu.edu.cn; Ihc9@hotmail.com ${ }^{\dagger}$ Shichong Qiao and Dongle Wu contributed equally to this work. ${ }^{2}$ Key Laboratory of Advanced Drug Preparation Technologies, Ministry of Education \& School of Pharmaceutical Sciences, Zhengzhou University, Zhengzhou 450001, Henan Province, PR China

'Department of Implant Dentistry, Shanghai Ninth People's Hospital, College of Stomatology, Shanghai Jiao Tong University School of Medicine, National Clinical Research Center for Oral Diseases, Shanghai Key Laboratory of Stomatology \& Shanghai Research Institute of Stomatology, Shanghai 200011, PR China
}

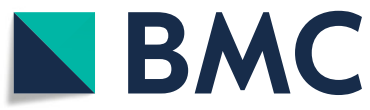

(c) The Author(s). 2020 Open Access This article is licensed under a Creative Commons Attribution 4.0 International License, which permits use, sharing, adaptation, distribution and reproduction in any medium or format, as long as you give appropriate credit to the original author(s) and the source, provide a link to the Creative Commons licence, and indicate if changes were made. The images or other third party material in this article are included in the article's Creative Commons licence, unless indicated otherwise in a credit line to the material. If material is not included in the article's Creative Commons licence and your intended use is not permitted by statutory regulation or exceeds the permitted use, you will need to obtain permission directly from the copyright holder. To view a copy of this licence, visit http://creativecommons.org/licenses/by/4.0/ The Creative Commons Public Domain Dedication waiver (http://creativecommons.org/publicdomain/zero/1.0/) applies to the data made available in this article, unless otherwise stated in a credit line to the data. soft and hard tissue around osseointegrated implants would result in the failure of dental implants known as inflammatory peri-implant disease [2-4]. Clinically, inflammatory peri-implant diseases are categorized into peri-implant mucositis and peri-implantitis $[5,6]$. Periimplant mucositis is defined as a reversible inflammatory reaction in the soft tissues surrounding a functioning implant, while peri-implantitis is described as inflammatory reactions involving supporting bone loss $[5,6]$. It has been estimated that the rate of implant-based and subject-based peri-implantitis are $9.25 \%$ and $19.83 \%$, 
respectively [7]. In another study, the prevalence of periimplantitis is up to $22 \%$ [8]. Therefore, understanding the mechanism that induces peri-implantitis is essential in current restorative dentistry, and might help to find preventive strategies for peri-implantitis and provide effective peri-implantitis treatment methods $[9,10]$.

It is generally accepted that peri-implantitis is a complex disease with multiple risk factors, and it initiates from a bacterial challenge and host overreaction [11, 12]. Much effort has been made to study the periimplant biofilm and its difference from periodontal biofilm [13], and reveal the bacterial colonization in healthy and diseased states [14]. The comparison between diseased peri-implant and healthy periodontal tissues has been applied [15]. Several potential microbial strains, such as Porphyromonas gingivalis and Prevotella intermedius, have been detected in oral biofilm at the periimplantitis sites. However, there is no clear consensus for core microbiota associated with peri-implantitis [16]. Traditional cultured strategies provide valuable information regarding known pathogens, but limited data on unculturable species and microbiota during periimplantitis development are available. Global investigation of microbiota variation during peri-implantitis development would make it possible to identify periimplantitis associated microbiota [17, 18], and highthroughput sequencing of $16 \mathrm{~S}$ rRNA gene technology provides a wealth of data pertaining to the differences between healthy and diseased implants $[19,20]$.

Experimental peri-implantitis animal models, such as mice and dogs, have been developed to study the mechanisms and development of marginal bone loss $[8,21]$. In previous animal studies, undisturbed peri-implant plaque accumulation often led to negligible or no marginal bone loss [22]. In order to obtain reliable periimplantitis animal models, sub-marginal ligatures of cotton, silk or other materials have been used to induce and speed up the peri-implantitis process [19, 23, 24]. Normally, sub-marginal ligation results in significant bone loss in a few weeks, and the peri-implantitis model can be established in a short time $[25,26]$. However, investigation of microbial dynamics during peri-implantitis model establishment had not been reported.

Here, the present study was designed to conduct a canine peri-implantitis model using sub-marginal ligatures method. The clinical parameters at different peri-implantitis phases were evaluated, and microbial variation associated with the severity of tissue destruction and inflammatory progression of experimental peri-implantitis were investigated. The core microbiota associated with peri-implantitis and their networks were analyzed, and the potential keystone microbial taxa associated with peri-implantitis were discussed.

\section{Results}

Establishment of peri-implantitis models

Eight teeth of each dog were successfully removed with minimal trauma (Supplementary Figure 1). After healing and recovery, the peri-implantitis induction was implemented. Overall, healing after tooth extraction and implant placement was uneventful at all surgical sites. Initial stability of each implant was confirmed after implant installation, and no implant loss was observed during the entire experimental period.

No visible inflammatory signs were observed before ligatures were applied at the implant sites (Phase T0) (Fig. 1 and Supplementary Figure 1). As expected, heavy plaque accumulation, bleeding on probing and probing depths increased following ligature placement, and samples at peri-implantitis timepoints were collected (Phase T1 to Phase T3). After each sampling, the implants were kept immobile by manually checking.

The disease severity around the inflammatory teeth were evaluated with heavy depth measurements (Fig. 2). The disease severity around the distobuccal, tongue side, and mesiobuccal increased during the peri-implantitis induction period (Phase T1-T2) and self-spontaneous recovery period (Phase T3). Meanwhile, the disease severity around buccal increased during the peri-implant inflammation period (Phase T1 and Phase T2), and the disease severity alleviated during the spontaneous recovery period (Phase T3). Taken together, teeth health deteriorated after peri-implantitis induction (Phase $\mathrm{T} 1$ to Phase T3) (Fig. 2).

\section{Diversity of the peri-implant sulcus samples at different phases}

The 24 peri-implant sulcus samples collected at Phase T0 to T3 were sequenced, and the data were used to indicate microbial diversity of these samples (Supplementary Figure 2). The operational taxonomic unit (OTU) numbers of these 24 samples were 206-445. The average OTU numbers of Phase T0 to T3 were 294, 337, 389, and 305 , respectively (Table 1 ). Only the OTU numbers between Phase T0 and Phase T2, as well as Phase T2 and Phase T3 displayed differences (Supplementary Table 1). The alpha parameters of Shannon_2, simpson, dominance and equitability at Phase T0 were higher than the respective parameters at Phase T1 to T3 (Table 1). Moreover, these four alpha parameters displayed differences or significant differences between the healthy state and the peri-implantitis period, including Phase T0 and Phase T1, Phase T0 and Phase T2, and Phase T0 and Phase T3 (Supplementary Table 1). However, these four alpha parameters displayed no differences between Phase T1 and Phase T2, Phase T1 and Phase T3, and Phase T2 and Phase T3 (Table 1 and Supplementary Table 1). 


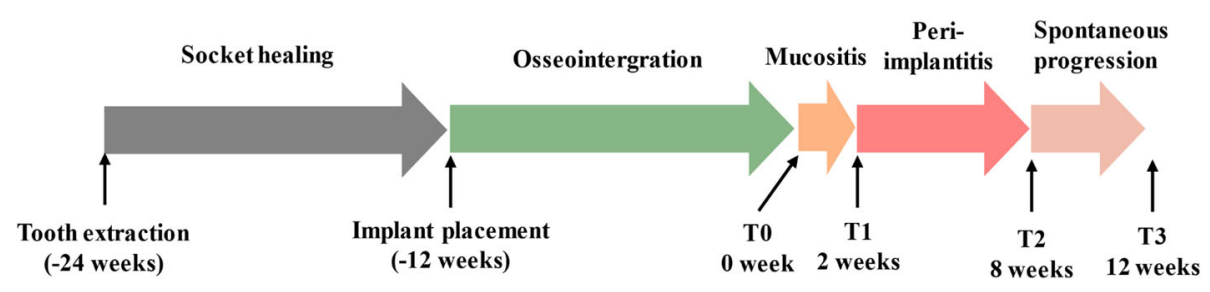

Fig. 1 Peri-implantitis development timeline and the sampling time points

\section{Microbiota analyses}

The microbial distribution of the same phases were similar (Supplementary Figure 3), while the microbial distribution of different phases harbored different phyla (Fig. 3a). At Phase T0 of the healthy state, the most dominant phyla were Firmicutes (68.1\%), Actinobacteria (11.7\%), and Bacteroidetes (9.4\%). At Phase T1 during the first 2 weeks of peri-implantitis, the most dominant phyla were Firmicutes (27.8\%), Bacteroidetes (35.8\%), and Spirochaetes (10.2\%). At Phase T2, the most dominant phyla were Firmicutes (39.9\%), Bacteroidetes (30.0\%), and Euryarchaeota (14.1\%). At Phase T3 of the selfspontaneous recovery period, the most dominant phyla were Firmicutes (30.7\%), Bacteroidetes (33.9\%), Spirochaetes (13.6\%), and Euryarchaeota (6.1\%). The Firmicutes, Bacteroidetes and Synergistetes distribution between Phase T0 and Phase T1 to T3 displayed significant differences. The Euryarchaeota distribution between Phase T0 and T2, T0 and T3, T1 and T2, and T1 and $\mathrm{T} 3$ displayed significant differences (Supplementary Table 2). Besides, a few other phyla displayed differences between different phases (Supplementary Table 2).
At the genus-level, microbial distribution at different phases changed (Fig. 3b). At Phase T0, the most dominant genera were Peptostreptococcus (43.5\%), Actinomyces (7\%), and Streptococcus (4.9\%). At Phase T1, the most dominant genera were Portostreptococcus (25.6\%), Treponema (10.0\%), and Fusobacterium (8.8\%). At Phase T2, the most dominant genera were Portostreptococcus (13.0\%), Peptostreptococcus (10.5\%), and Methanobrevibacter (5.7\%). At Phase T3, the most dominant genera were Portostreptococcus (20.0\%), Treponema (13.4\%), and Portostreptococcus (10.9\%). Some genera, including Peptostreptococcus, Porphyromonas, Treponema and Fretibacterium, displayed significant differences between Phase T0 and Phase T1 to T3. The Methanobrevibacter displayed significant differences between $\mathrm{T} 0$ and $\mathrm{T} 2, \mathrm{~T} 0$ and $\mathrm{T} 3, \mathrm{~T} 1$ and $\mathrm{T} 2$, and $\mathrm{T} 1$ and T3 (Supplementary Table 3). No significant differences of Bacteroides and Streptococcus between Phase T0 and Phase T1 to Phase T3 were observed.

\section{The dominant OTUs in the microbiota}

Most OTUs with average compositions $>1 \%$ in the four phases had $>97 \%$ identity with known isolates (Table 2 ).

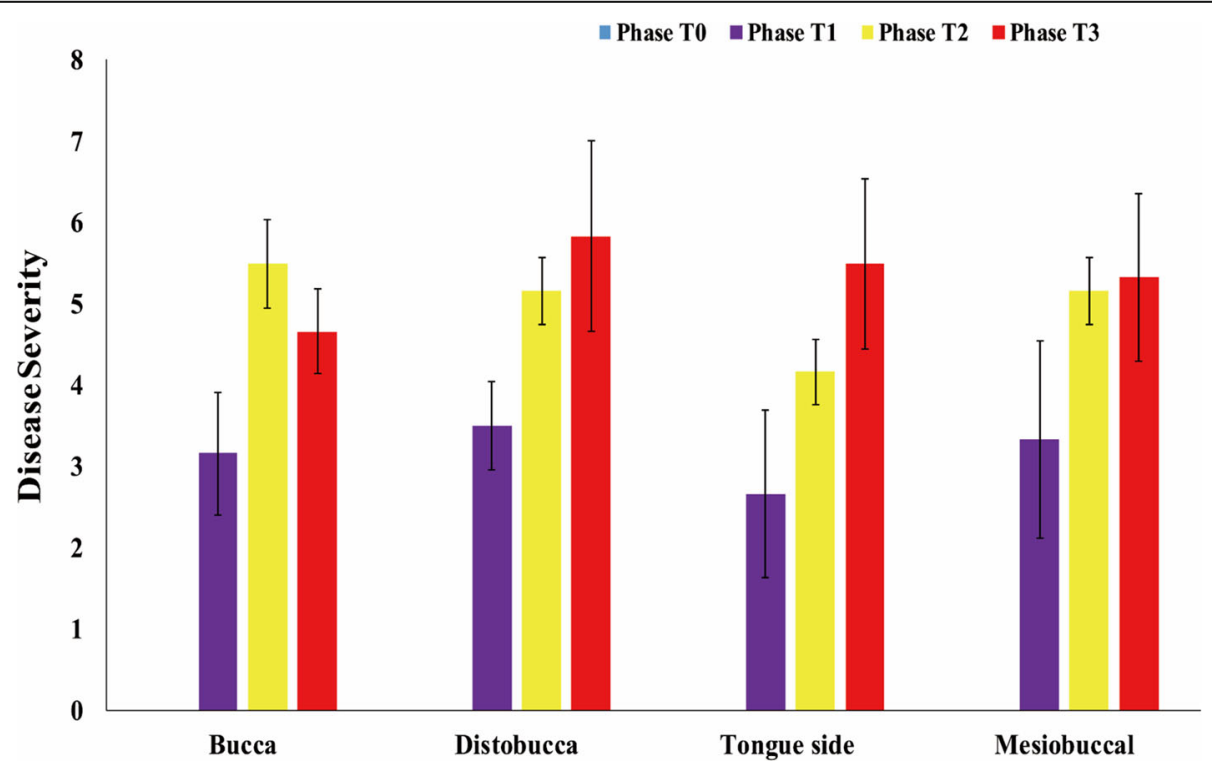

Fig. 2 Disease severity of the bucca, distobucca, tongue side and mesiobuccal of the implants during Peri-implantitis development. The error bars represent the standard deviation of six replicates of each site around the implant 
Table 1 Average alpha diversity parameters during Peri-implantitis development of the dog teeth

\begin{tabular}{lllllll}
\hline & Richness & Chao1 & Shannon_2 & Simpson & Dominance & Equitability \\
\hline Phase T0 & $294 \pm 78$ & $295 \pm 78$ & $4 \pm 0.8$ & $0.2 \pm 0.1$ & $0.8 \pm 0.1$ & $0.5 \pm 0.1$ \\
Phase T1 & $337 \pm 76$ & $339 \pm 75$ & $5.3 \pm 0.5$ & $0.07 \pm 0.03$ & $0.9 \pm 0.03$ & $0.6 \pm 0.08$ \\
Phase T2 & $389 \pm 34$ & $390 \pm 34$ & $5.6 \pm 0.4$ & $0.05 \pm 0.02$ & $1 \pm 0.02$ & $0.7 \pm 0.04$ \\
Phase T3 & $305 \pm 78$ & $307 \pm 76$ & $5.1 \pm 0.4$ & $0.06 \pm 0.02$ & $0.9 \pm 0.02$ & $0.6 \pm 0.07$ \\
\hline
\end{tabular}
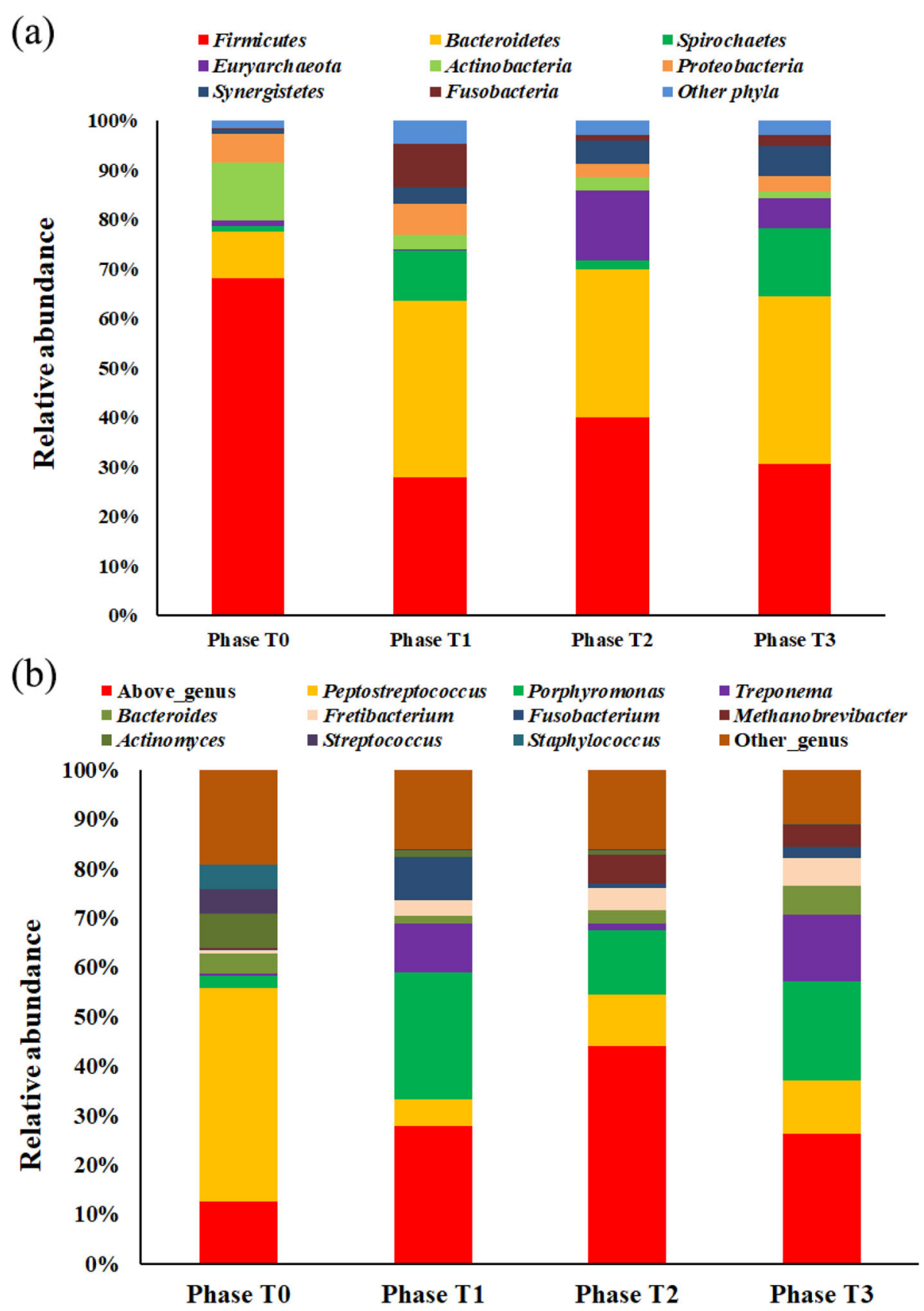

Fig. 3 Microbial compositions of the implant samples during Peri-implantitis development at phylum-level (a) and genus-level (b) 
Table 2 The distribution of dominant OTUs identified during Peri-implantitis development and the 16S rRNA gene fragments of their closest isolates and uncultured bacteria

\begin{tabular}{|c|c|c|c|c|c|c|c|c|}
\hline & Closest isolates & Identity & Closest uncultured (nr/nt) & Identity & $\begin{array}{l}\text { Phase } \\
\text { T0 }\end{array}$ & $\begin{array}{l}\text { Phase } \\
\text { T1 }\end{array}$ & $\begin{array}{l}\text { Phase } \\
\text { T2 }\end{array}$ & $\begin{array}{l}\text { Phase } \\
\text { T3 }\end{array}$ \\
\hline $\begin{array}{l}\text { OTU } \\
1\end{array}$ & Peptostreptococcus canis (NR_117641.1) & $99.50 \%$ & Uncultured bacterium (JQ192915.1) & $100 \%$ & $43.46 \%$ & $5.48 \%$ & $10.50 \%$ & $10.89 \%$ \\
\hline $\begin{array}{l}\mathrm{OTU}_{-} \\
3\end{array}$ & Porphyromonas macacae (NR_025908.1) & $97.88 \%$ & Uncultured bacterium (KX437329.1) & $100 \%$ & $0.19 \%$ & $6.29 \%$ & $0.95 \%$ & $10.98 \%$ \\
\hline $\begin{array}{l}\mathrm{OTU}_{-} \\
7\end{array}$ & $\begin{array}{l}\text { Porphyromonas endodontalis (NR_ } \\
\text { 113085.1) }\end{array}$ & $94.37 \%$ & $\begin{array}{l}\text { Uncultured Porphyromonas sp. canine } \\
\text { oral (JN713348.1) }\end{array}$ & $99.30 \%$ & $0.12 \%$ & $0.48 \%$ & $5.43 \%$ & $6.71 \%$ \\
\hline $\begin{array}{l}\mathrm{OTU}_{-} \\
13\end{array}$ & Fretibacterium fastidiosum (NR_108538.1) & $95.82 \%$ & $\begin{array}{l}\text { Uncultured Fretibacterium sp. Feline } \\
\text { (KM462182.1) }\end{array}$ & $100 \%$ & $0.58 \%$ & $3.05 \%$ & $3.99 \%$ & $4.66 \%$ \\
\hline $\begin{array}{l}\mathrm{OTU}_{-} \\
5\end{array}$ & Bacteroides pyogenes (NR_113048.1) & $100 \%$ & Uncultured Bacteroides (MH755473.1) & $100 \%$ & $3.49 \%$ & $0.61 \%$ & $2.32 \%$ & $5.30 \%$ \\
\hline $\begin{array}{l}\text { OTU } \\
9\end{array}$ & Fusobacterium simiae (NR_113318.1) & 99.51 & $\begin{array}{l}\text { Uncultured Fusobacterium sp. canine } \\
\text { (JN713401.1) }\end{array}$ & $100 \%$ & $0.07 \%$ & $8.22 \%$ & $0.79 \%$ & $2.07 \%$ \\
\hline $\begin{array}{l}\text { OTU } \\
8\end{array}$ & Porphyromonas gulae (NR_113088.1) & $99.53 \%$ & Uncultured bacterium (HM328336.1) & $99.76 \%$ & $0.29 \%$ & $6.92 \%$ & $2.65 \%$ & $0.88 \%$ \\
\hline $\begin{array}{l}\mathrm{OTU}_{-} \\
4\end{array}$ & Methanobrevibacter oralis (LN898260.1) & $100 \%$ & $\begin{array}{l}\text { Uncultured Methanobrevibacter } \\
\text { (JN052095.1) }\end{array}$ & $100 \%$ & $0.23 \%$ & $0.09 \%$ & $5.68 \%$ & $4.53 \%$ \\
\hline $\begin{array}{l}\mathrm{OTU}_{-} \\
2\end{array}$ & $\begin{array}{l}\text { Methanimicrococcus blatticola } \\
\text { (JQ268014.1) }\end{array}$ & $98.97 \%$ & $\begin{array}{l}\text { Uncultured Methanimicrococcus } \\
\text { (LT624863.1) }\end{array}$ & $97.42 \%$ & $0.24 \%$ & $0.09 \%$ & $8.23 \%$ & $1.55 \%$ \\
\hline $\begin{array}{l}\text { OTU } \\
19\end{array}$ & Treponema denticola (NR_074582.1) & $97.91 \%$ & Uncutlured Treponema (JN713361.1) & $100 \%$ & $0.09 \%$ & $3.37 \%$ & $0.79 \%$ & $4.89 \%$ \\
\hline $\begin{array}{l}\mathrm{OTU}_{-} \\
10\end{array}$ & $\begin{array}{l}\text { Porphyromonas cangingivalis (NR_ } \\
\text { 113080.1) }\end{array}$ & $100 \%$ & Uncultured bacterium (JF241087.1) & $100 \%$ & $0.85 \%$ & $6.38 \%$ & $1.72 \%$ & $0.10 \%$ \\
\hline $\begin{array}{l}\mathrm{OTU}_{-} \\
6\end{array}$ & $\begin{array}{l}\text { Intestinimonas butyriciproduens (NR_ } \\
\text { 118554.1) }\end{array}$ & $94.1 \%$ & $\begin{array}{l}\text { Uncultured Clostridiales bacterium } \\
\text { (JN713380.1) }\end{array}$ & $99.75 \%$ & $0.40 \%$ & $0.17 \%$ & $5.41 \%$ & $2.56 \%$ \\
\hline $\begin{array}{l}\mathrm{OTU}_{-} \\
14\end{array}$ & Treponema sp. PT8 (AM980447.1) & $100 \%$ & Uncultured Treponema sp. (GQ424168.1) & $99.77 \%$ & $0.05 \%$ & $2.75 \%$ & $0.06 \%$ & $4.36 \%$ \\
\hline $\begin{array}{l}\mathrm{OTU}_{-} \\
37\end{array}$ & $\begin{array}{l}\text { Odoribacter denticanis strain (NR_ } \\
\text { 042977.1) }\end{array}$ & $99.76 \%$ & $\begin{array}{l}\text { Odoribacter denticanis canine oral } \\
\text { (JN713247.1) }\end{array}$ & $99.76 \%$ & $0.83 \%$ & $1.28 \%$ & $2.29 \%$ & $1.79 \%$ \\
\hline $\begin{array}{l}\mathrm{OTU}_{-} \\
16\end{array}$ & Olivibacter sitiensis (NR_043805.1) & $84.15 \%$ & $\begin{array}{l}\text { Uncultured Bacteroidia bacterium } \\
\text { (KM462114.1) }\end{array}$ & $100 \%$ & $0.07 \%$ & $0.04 \%$ & $3.87 \%$ & $1.81 \%$ \\
\hline $\begin{array}{l}\mathrm{OTU}_{-} \\
17\end{array}$ & $\begin{array}{l}\text { Staphylococcus intermedius NCTC } 11048 \\
\text { (NR_113351.1) }\end{array}$ & $100 \%$ & $\begin{array}{l}\text { Uncultured Staphylococcus sp. strain } \\
\text { (JX482520.1) }\end{array}$ & $100 \%$ & $4.94 \%$ & $0.02 \%$ & $0.02 \%$ & $0.07 \%$ \\
\hline $\begin{array}{l}\mathrm{OTU}_{-} \\
15\end{array}$ & Metaprevotella massiliensis (NR_147377.1) & $90.59 \%$ & $\begin{array}{l}\text { Prevotella sp. canine oral taxon } \\
\text { (JN713360.1) }\end{array}$ & $100 \%$ & $0.09 \%$ & $0.45 \%$ & $3.52 \%$ & $0.91 \%$ \\
\hline $\begin{array}{l}\mathrm{OTU}_{-} \\
11\end{array}$ & $\begin{array}{l}\text { Porphyromonas crevioricanis (NR_ } \\
\text { 104834.1) }\end{array}$ & $100 \%$ & Uncultured bacterium (JF17468.1) & $100 \%$ & $0.04 \%$ & $2.47 \%$ & $1.24 \%$ & $1.16 \%$ \\
\hline $\begin{array}{l}\mathrm{OTU}_{-} \\
47\end{array}$ & Schaalia canis (NR_025366.1) & $98.34 \%$ & Uncultured bacterium (JF223833.1) & $100 \%$ & $3.76 \%$ & $0.51 \%$ & $0.35 \%$ & $0.02 \%$ \\
\hline $\begin{array}{l}\mathrm{OTU}_{-} \\
26\end{array}$ & $\begin{array}{l}\text { Intestinimonas butyriciproducens (NR } \\
\text { 118554.1) }\end{array}$ & $94.84 \%$ & $\begin{array}{l}\text { Uncultured Ruminococcaceae bacterium } \\
\text { (MH572181.1) }\end{array}$ & $100 \%$ & $0.08 \%$ & $0.94 \%$ & $1.66 \%$ & $1.77 \%$ \\
\hline $\begin{array}{l}\mathrm{OTU}_{-} \\
12\end{array}$ & Labilibacter aurantiacus (NR_156071.1) & $85.31 \%$ & Uncultured bacterium (KI874155.1) & $92.47 \%$ & $0.12 \%$ & $1.71 \%$ & $1.40 \%$ & $1.10 \%$ \\
\hline $\begin{array}{l}\mathrm{OTU}_{-} \\
30\end{array}$ & Filifactor villosus (NR_041928.1) & $99.01 \%$ & Filifactor sp. Feline (KM462053.1) & $100 \%$ & $0.19 \%$ & $2.17 \%$ & $1.09 \%$ & $0.59 \%$ \\
\hline
\end{tabular}

Besides, OTU distribution at different phases displayed difference or significant difference (Supplementary Table 4). OTU_1, which composes $43.46 \%$ at Phase T0, might be one Peptostreptococcus canis strain isolated from subgingival plaque from canine oral cavity [27]. Compared with Phase T0, P. canis composition decreased to $5.5 \%$ at Phase $\mathrm{T} 1,10.5 \%$ at Phase $\mathrm{T} 2$, and
$10.9 \%$ at Phase T3. The compositions of OTU_3 which might be Porphyromonas macacae increased from < $0.2 \%$ at Phase T0 to $6.3 \%$ at Phase T1, $1 \%$ at Phase T2, and $11 \%$ at Phase T3 [28]. OTU_7, which was assigned to the genus of Porphyromonas, composed $0.12 \%$ at Phase T0, but increased to $0.48 \%$ at Phase T1, 5.43\% at Phase T2, and $6.71 \%$ at Phase T3. OTU_13, which was 
assigned to the genus of Fretibacterium, composed only $0.58 \%$ at Phase T0, but it increased to $>3 \%$ at Phase T1 to T3. In particular, some methanogens, such as OTU_2 and OTU_4, composed $<0.25 \%$ at Phase T0. The composition of OTU_2 and OTU_4 increased to $13.91 \%$ at phase T2, and their composition decreased to $6.08 \%$ at Phase T3. A few other dominant OTUs were assigned to the typical oral microbiota or clinical pathogens, and the compositions of clinical pathogens increased (Table 2). For example, OTU_19 was assigned to teeth infection associated bacteria Treponema denticola [29, 30]. OTU_ 19 composition at Phase T0 was $0.09 \%$, and its composition increased to $3.37 \%$ at Phase T1, $0.79 \%$ at Phase T2, and $4.89 \%$ at Phase T3.

\section{Microbial similarity of the samples at different phases}

The microbial composition was used to compare samples at different phases. The NMDS result based on Bray Cutis distance showed that the six samples at Phase T0 were clustered and were obviously different from other peri-implantitis samples. Phase T1 samples were clustered, and were different from other samples. Moreover, twelve samples at Phase T2 and Phase T3 were clustered, and were different from samples at Phase T0 and Phase T1 (Fig. 4a). The UPGMA tree based on Bray Cutis distance also showed that samples at different phases were clustered. In particular, the six samples at Phase T0 were clustered, and another eighteen samples at Phase T1 to T3 were clustered (Fig. 4b). In addition, the distance between samples at Phase T2 and Phase T3 was shorter than the distance between samples at Phase T1 and Phase T2 or Phase T1 and Phase T3. In all, the UPGMA result was in accordance with the NMDS result that the healthy state Phase T0 samples were divergent from the diseased samples at Phase T1 to Phase T3.

\section{Network analyses of the microbiota}

The correlations of all the samples were calculated, and only the dominant OTUs $(>0.5 \%)$ with strong relationship ( $\rho>0.6$ and $P<0.05$ ) were used for network analyses [31, 32]. The co-occurrence network contained 37 nodes (OTUs) and 208 edges. The compositions of these OTUs in the 24 samples were between $0.54 \%$ and $4.6 \%$ (Fig. 5). Among the 208 edges, only one edge between OTU_18 and OTU_28 indicated negative correlation. The mean edge per node was 11 , and the eccentricity of the node was 3.6. The average shortest path length was 2.1 with a closeness centrality of 0.5 . The average radiality of the node was 0.8 , and the topological coefficient was 0.5 .

Among the 37 nodes (OTUs), 13 of them were assigned to Bacteroidetes, and 12 of them were assigned to Firmicutes. The composition of Bacteroidetes and Firmicutes OTUs of the 37 OTUs was $67.57 \%$. Interestingly, Bacteroidetes and Firmicutes were the two most dominant phyla of the 24 samples, and composed $68.89 \%$ of

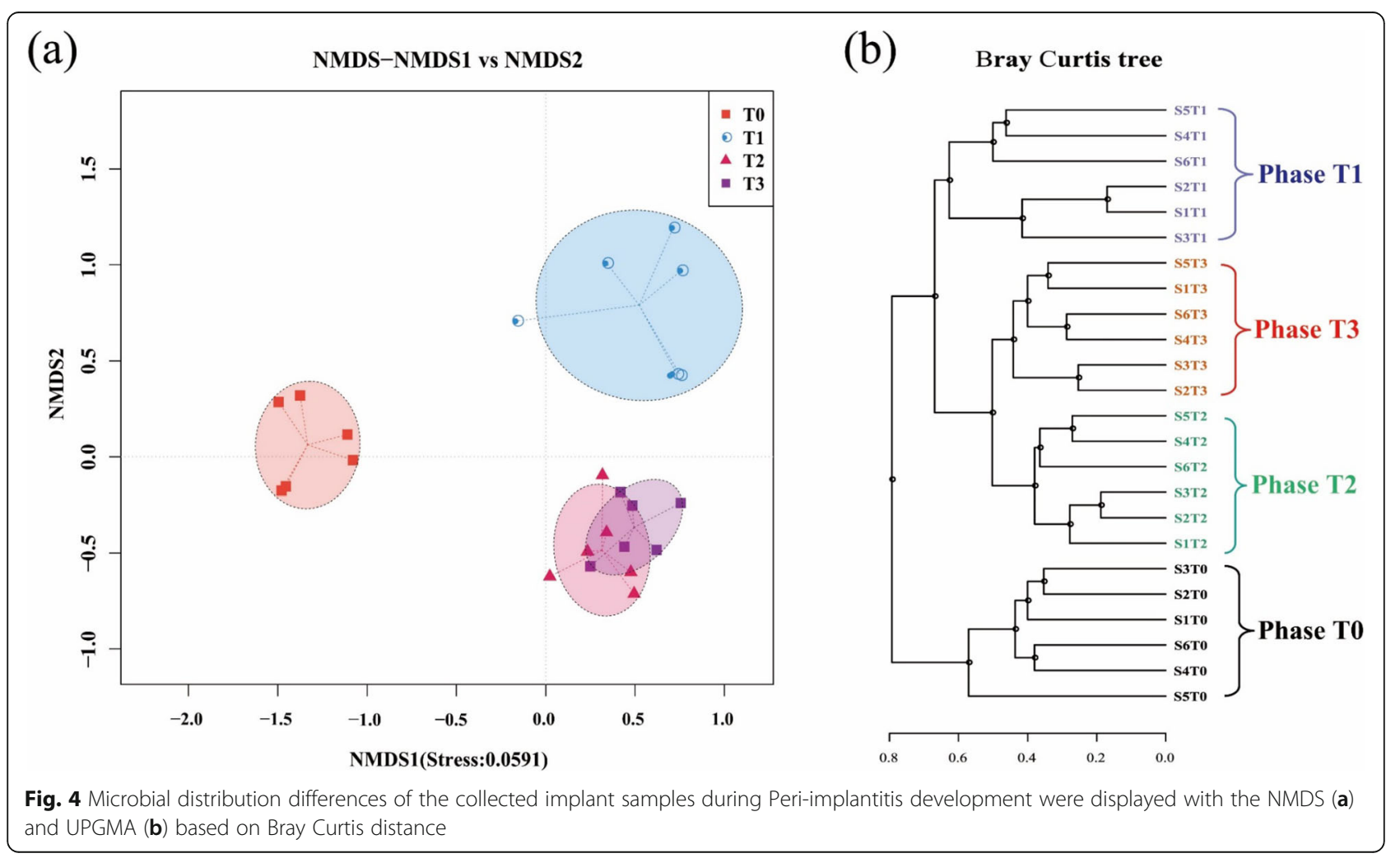




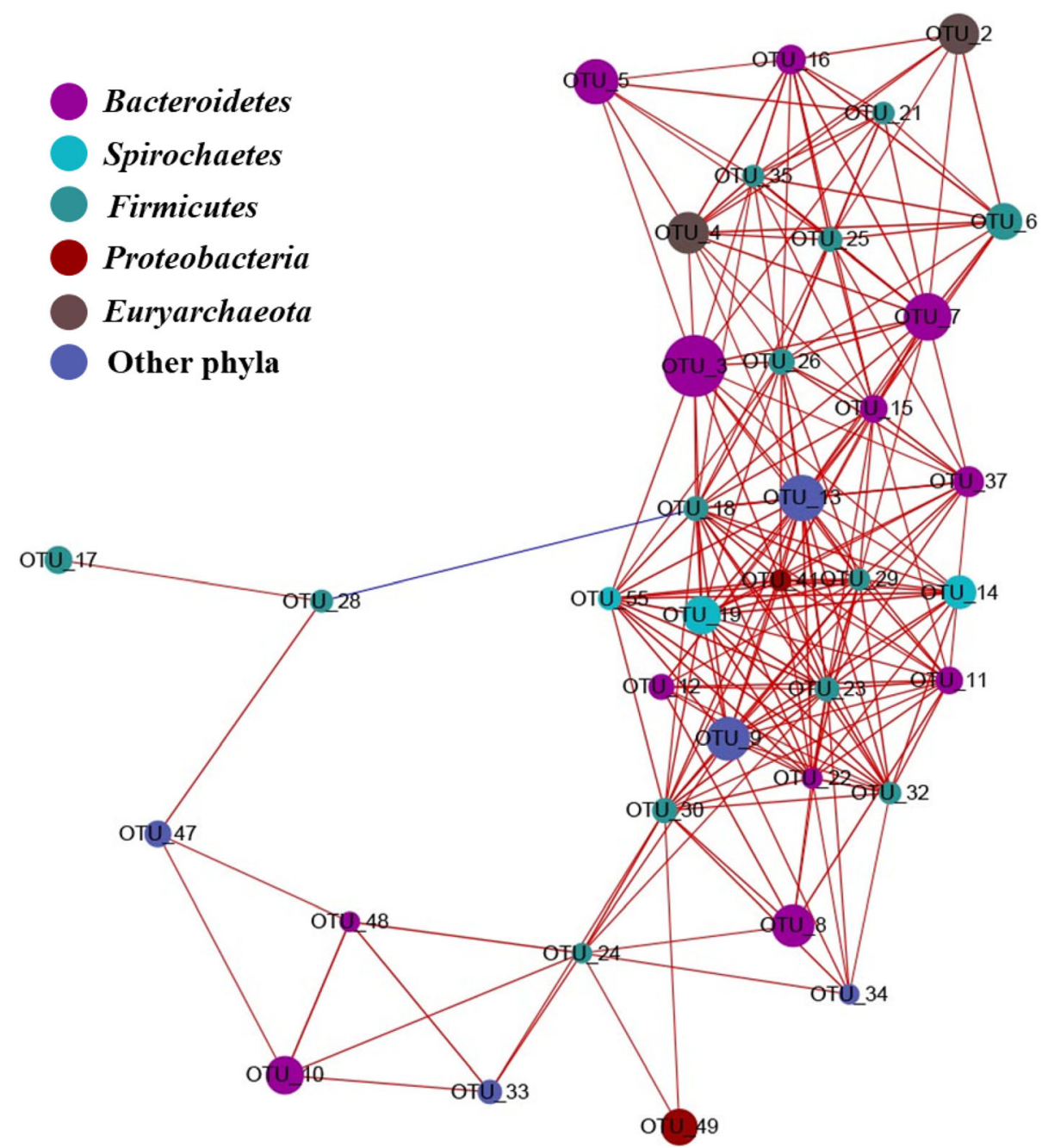

Fig. 5 Network of co-occurring dominant OTUs (with relative composition $>0.5 \%$ ) based on correlation analysis. The selection standards for strong correlation are Spearman's $\rho>0.6$, and significant correlation with $P<0.05$. The size of each node is proportional to the relative abundance; the thickness of each connection between two nodes (edge) is proportional to the value of Spearman's correlation coefficients. The line between two nodes in blue shows negative correlation between the two nodes; the line between two nodes in red shows positive correlation between the two nodes

the microbiota (Fig. 3a). All these 37 OTUs were among the top39 OTUs of the whole microbiota. However, the most abundant OTU in the microbiota, OTU_1, was not in the network. Besides, OTU_ 122, which composed $12.34 \%$ of one sample and had an average composition in the 24 samples of $0.56 \%$, was not in the network. Based on the connection in the network and average compositions, OTU_3, OTU_7, OTU_13, OTU_18, OTU_23, OTU_29, OTU_26, OTU_41, and OTU_30 were identified as the keystone taxa (Fig. 5). Additionally, the compositions of all these nine OTUs at Phase $\mathrm{T} 0$ were lower than their corresponding compositions in Phase T1 to Phase T3. Among the nine keystone taxa, four of them (OTU_18, OTU_23, OTU_26, OTU_30) were assigned to Firmicutes, three of them (OTU_3, OTU_7, OTU_29) were assigned to Bacteroidetes, OTU_
13 was assigned to Synergistetes, and OTU_41 was assigned to Proteobacteria.

\section{Discussion}

Peri-implantitis strongly affects adjacent tissues, resulting in losing the function of the osseointegrated implant $[7,8]$. Peri-implantitis is caused by microbial infection, but the associated pathogenic microbes are unclear [9]. The microbial distributions between the healthy dental sites and peri-implantitis sites in subjects with chronic periodontitis had been conducted, showing diseased implants and corresponding periodontally healthy sites harbored different microbiota [33]. However, global microbial variation during peri-implantitis development was unknown. The comparison between human healthy and diseased peri-implant sites had been tried, but the 
human data might be affected by gender, antibiotic intake, or age [34]. The animal model can provide periimplantitis samples with the same criteria [35]. In this study, the clinical parameters at different phases during peri-implantitis setup and progression in a canine model were collected, showing that the disease severity increased during peri-implantitis development (Fig. 2). Moreover, the microbiota changed dramatically during the shift from healthy status to peri-implantitis status.

The clinical parameters showed that the disease severity increased and the peri-implantitis model was successfully built, indicating cotton ligature around dental implant is one effective strategy for peri-implantitis induction [19]. During peri-implantitis development, microbial richness and diversity increased from the healthy phase to diseased phases (Table 1), suggesting high microbial richness and diversity were associated with severe infection. These results were different from previous results that high microbial richness was associated with healthy samples [33]. However, the increased richness and diversity in canine peri-implantitis development complements earlier study results that periimplantitis sites exhibited higher bacterial counts compared to healthy sites [36]. The increased microbial species during in peri-implantitis development might not only derive from the original dental implant sites, but also from the oral microbiome or environmental microbiome [14]. The decreased microbial richness and diversity from Phase T2 to Phase T3 (Table 1) suggested that the host self-spontaneous progression recovery system functioned and the health status improved after removal of the ligatures.

The human oral microbiome database (HOMD) collects bacterial species distributed in the oral region and the upper respiratory tracts, and the dominant phyla were Actinobacteria, Firmicutes, and Proteobacteria [37]. Different from the microbial collection in HOMD, the main phyla in the healthy implants were Firmicutes (51.9\%), Bacteroidetes (18.5\%), Fusobacteria (11.1\%), and Proteobacteria (7.4\%), while the dominant phyla in periimplantitis were Firmicutes (30.6\%), Bacteroidetes (40.3\%), Fusobacteria (13.9\%), and Proteobacteria (5.6\%) [15]. The decrease of Firmicutes and increase of Bacteroidetes in human peri-implantitis were the same as the microbiota variation during peri-implantitis development in this study [34], showing that phyla variation might be a common phenomenon during periimplantitis development in human and animals. The Synergistetes and Spirochaetes compositions of human peri-implantitis sites were higher than that of human healthy sites [34, 36], and their compositions increased during peri-implantitis development (Fig. 3). Compared with healthy dental implant sites, $T$. denticola were often detected in peri-implantitis sites [36]. In this study, more
T. denticola were detected at Phase T2 and T3 (Table 2 ). The results suggested a canine model can imitate human peri-implantitis, which might be useful in future human peri-implantitis therapeutic studies.

Peri-implantitis has been successfully induced by oral infection with $P$. gingivalis in mice, and $P$. gingivalis has often been identified at peri-implantitis sites [12, 38, 39]. OTU_3 and OTU_7 assigned to Porphyromonas were identified in this study, and the compositions of OTU_3 and OTU_7 were higher at the diseased phases (Phase T1 to Phase T3) than at the healthy phase (Phase T0), showing Porphytomonas might be associated with periimplantitis. OTU_3 and OTU_7 were identified as keystone taxa, suggesting these two strains might be one potential peri-implantitis cause [10]. The compositions of methanogens were higher at Phase T2 and Phase T3, but they were not keystone taxa, indicating methanogens might not be an indicator of peri-implantitis development [40]. Prevotella nigrescens was also identified to be associated with peri-implantitis lesion [12, 41], but no Prevotella was identified in oral peri-implantitis microbiome in this study (Table 2). Though the microbiota between humans and animals were slightly different at species-level, establishment of animal oral models might give insights into microorganisms associated with human oral diseases in the future.

\section{Conclusion}

In summary, we successfully established an experimental peri-implantitis animal model using ligature-induced method, and the increased severity of inflammation is consistent with the progression of the periimplantitis disease as we expected. The investigation of the microbial variation during peri-implantitis development identified several OTUs associated with periimplantitis. This study provides insight into the periimplantitis microbiome and deepens our understanding of keystone taxa during peri-implantitis development.

\section{Methods \\ Animals}

The animal experiment had been reviewed and approved by the Ethics Committee for Animal Research in Shanghai 9th People's Hospital, Shanghai Jiao Tong University. Three two-year-old male beagles weighing $10-15 \mathrm{~kg}$ were bought from Shanghai Jiagan Biotech Co. Ltd. and used for the peri-implantitis experiment in this study. The dogs were housed individually and fed a soft diet at the Animal Research Center of Shanghai 9th People's Hospital, School of Medicine, Shanghai Jiao Tong University, Shanghai, China. All the dogs had fully erupted permanent dentition and good general health before the experiment. The dogs were sacrificed in enthanasia by 
intravenous administration of a lethal pentoharbital sodium dose of $120 \mathrm{mg} / \mathrm{kg}$ weight.

The remaining teeth were cleaned twice a week with $0.12 \%$ chlorhexidine and soft toothbrush. All surgical procedures were performed under general anesthesia by sedation with $2 \%$ xylazine hydrochloride and ketamine hydrochloride/zolazepam hydrochloride. The surgical sites were locally anesthetized using $2 \%$ lidocaine hydrochloride with 1:100,000 epinephrine. The surgical site was shaved and cleaned with chlorhexidine ethanol solution $0.5 \mathrm{mg} / \mathrm{ml}$, and covered with a sterile surgical drape. The animals were made to fast for $12 \mathrm{~h}$ preoperatively, but allowed to drink water ad libitum.

\section{Teeth removal and implant placement}

All the premolars (M1-M4) in the mandible (eight teeth of each dog) were extracted bilaterally from the three dogs using a minimally invasive approach (Supplementary Figure 1). After a healing period of 12 weeks, three implants $(3.0 \mathrm{~mm}$ in diameter and $9.0 \mathrm{~mm}$ in length, Astra, Switzerland) were inserted at the extraction sites of both sides. Mechanical cleaning was performed to remove supragingival calculus around natural teeth 1week pre-operation. All implants were left to heal in a submerged position for another 12 weeks, and the second-stage surgery was conducted to replace cover screws with healing abutments (width: $4 \mathrm{~mm}$, height: 4 $\mathrm{mm}$ ) (Phase T0) (Fig. 1). No plaque control procedures were performed after ligature placement.

\section{Induction of peri-implantitis and sample collection}

A cotton ligature was ligated around each implant fixture below the healing abutment to allow for plaque accumulation and inflammatory infection $[19,42]$. Two weeks later, a clinical and radiologic examination indicated that the peri-implant mucositis was established, and the samples were collected (Phase T1). Meanwhile, additional ligation was performed below the preexisting ligatures every 2 weeks, and the samples were collected after another 6 weeks (Phase T2), while radiography showed significant bone loss. Then the ligatures were removed, and the inflammation sites were left alone for self-spontaneous progression recovery. Four weeks later, samples were collected (Phase T3) (Fig. 1).

During sample collection, the sampling sites were dried with an air pistol, and samples of peri-implant sulcular fluid (PISF) were collected [39]. Sampling sites were exposed from moisture after supra-mucosal plaque was removed. Filter strips $(2 \mathrm{~mm} \times 10 \mathrm{~mm})$ were inserted into the bottom of the peri-implant sulcus with a mild resistance at the buccomesial and buccodistal aspects of peri-implant sulcus for $30 \mathrm{~s}$. All the collected strips were stored at $-80{ }^{\circ} \mathrm{C}$ before use.

\section{Clinical and radiographic examination}

Clinical measurements were taken at the mesial, buccal, distal, and lingual sites of each implant using a UNC15 probe (Hu-Friedy, Chicago, IL, USA). The peri-implant probing depths (PD) and the relative clinical attachment levels (rCAL) in $\mathrm{mm}$ were measured. In addition, the Modified Plaque Index (mPI) was measured along the mucosal margin and recorded as presence (1) or absence (0); the bleeding on probing (BOP) was measured $15 \mathrm{~s}$ after probing and recorded as presence (1) or absence (0).

These measurements were performed by two examiners after a calibration exercise demonstrating $95.3 \%$ concordance within $1 \mathrm{~mm}$ for measurements of PD. The disease severity was indicated based on the measured clinical parameters.

Intra-oral radiographs of all the implant sites were taken using paralleling technique combined with long cone (Rinn XCP, Dentsply, Elgin, IL, USA).

\section{DNA extraction and sequencing}

DNA were extracted from the collected strip samples using DNeasy Kit (Cat No./ID: 47014, Qiagen, Germany). DNA of each sample was diluted to proper concentration for gene amplification of 16S rRNA gene V3-V4 regions [43]. The Phanta Max Super-Fidelity DNA Polymerase (Vazyme Biotech, Nanjing, China) was used for $16 \mathrm{~S}$ rRNA gene fragment amplification following the manufacturer's procedure, and the amplification cycles were 27. Further sequencing was implemented by DeepLab Co., Ltd. (Shanghai, China).

\section{$16 \mathrm{~S}$ rRNA gene analyses}

USEARCH software with default parameters were used to analyze the fastq files [44]. The USEARCH UPARSE was used to classify the obtained clean reads into operational taxonomic unit (OTU) based on 97\% identity, and UPARSE analysis pipeline was applied to remove chimeric reads [43, 45]. USEARCH was used to analyze the phylogenetic classification of the obtained 16S rRNA gene fragment sequences, and RDP training set (version v16) with a confidence threshold of 0.8 was used as the reference database [46]. In addition, USEARCH was further used for the $\alpha$ and $\beta$ diversity analyses [47]. The unweighted pair-group method with arithmetic means (UPGMA) and non-metric multidimensional scaling (NMDS) based on Bray Curtis were analyzed by $R$ software [47].

The co-occurrence network analysis strategy was carried out to explore co-occurrence of the abundant OTUs in the microbiota [48]. The correlation between OTUs was calculated with spearman's method with pairwise distance. To identify keystone taxa, only strong correlation between OTUs' relationship with $\rho>0.6$, and significant correlation with $P<0.05$ was kept. Finally, only OTUs with relative composition $>0.5 \%$ were used for further co-occurrence analysis and network construction using Cytoscape [32, 49]. 


\section{Supplementary information}

Supplementary information accompanies this paper at https://doi.org/10. 1186/s12866-020-01982-6.

\section{Additional file 1}

\section{Acknowledgments}

We thank Dr. Boyang Ji at Chalmers University of Technology and Dr. Xiaogang Xiang at Shanghai Jiao Tong University School of Medicine for revising English writing of this manuscript. We sincerely thank Lei Zhang from DeepLab Co., Ltd. (Shanghai, China) for bioinformatic help. We sincerely thank for the support from Shanghai Zammax Biotech Co., Ltd.

\section{Authors' contributions}

Conception and design: SCQ, YW, HL; Data acquisition: SCQ, YW, DW, SJQ, YZ, JS; Analyses and interpretation of the data: SCQ, YW, MW; Drafting of the article: YW, CQ, DW, MW. All authors have read and approved the manuscript.

\section{Funding}

This study has been supported by Science and Technology Commission of Shanghai Municipality $(16441903600,20 S 31906100)$, the National Natural Science Foundation of China (81600902), Clinical Research Plan of SHDC 16CR3033A, and the Project of Biobank (NO. YBKA201906) from Shanghai Ninth People's Hospital, Shanghai Jiao Tong University School of Medicine.

\section{Availability of data and materials}

All the obtained data were deposited in Genbank database, and the accession numbers of the samples were SRR10695872-SRR10695895.

\section{Ethics approval and consent to participate}

The animal experiment had been reviewed and approved by the Ethics Committee for Animal Research in Shanghai 9th People's Hospital, Shanghai Jiao Tong University.

\section{Competing interests}

The authors declare that they have no competing interests.

Received: 16 July 2020 Accepted: 18 September 2020

Published online: 29 September 2020

\section{References}

1. Peres MA, Macpherson LMD, Weyant RJ, Daly B, Venturelli R, Mathur MR Listl S, Celeste RK, Guarnizo-Herreno CC, Kearns C, et al. Oral diseases: a global public health challenge. Lancet. 2019;394(10194):249-60.

2. Manor $Y$, Oubaid S, Mardinger $O$, Chaushu G, Nissan J. Characteristics of early versus late implant failure: a retrospective study. J Oral Maxil Surg. 2009:67(12):2649-52

3. Insua A, Monje A, Wang HL, Miron RJ. Basis of bone metabolism around dental implants during osseointegration and peri-implant bone loss. J Biomed Mater Res A. 2017;105(7):2075-89.

4. Derks J, Schaller D, Håkansson J, Wennström JL, Tomasi C, Berglundh T. Periimplantitis - onset and pattern of progression. J Clin Periodontol. 2016; 43(4):383-8

5. Berglundh T, Armitage G, Araujo MG, Avila-Ortiz G, Blanco J, Camargo PM, Chen S, Cochran D, Derks J, Figuero E, et al. Peri-implant diseases and conditions: Consensus report of workgroup 4 of the 2017 World workshop on the classification of periodontal and Peri-implant diseases and conditions. J Periodontol. 2018:89(Suppl 1):S313-8.

6. Papapanou PN, Sanz M, Buduneli N, Dietrich T, Feres M, Fine DH, Flemmig TF, Garcia R, Giannobile W, Graziani F, et al. Periodontitis: Consensus report of workgroup 2 of the 2017 World workshop on the classification of periodontal and Peri-implant diseases and conditions. J Periodontol. 2018; 89(Suppl 1):S173-82.

7. Lee CT, Huang YW, Zhu L, Weltman R. Prevalences of peri-implantitis and peri-implant mucositis: systematic review and meta-analysis. J Dent. 2017;62: $1-12$.

8. Derks J, Tomasi C. Peri-implant health and disease. A systematic review of current epidemiology. J Clin Periodontol. 2015;42(Suppl 16):S158-71.
9. Salvi GE, Cosgarea R, Sculean A. Prevalence and mechanisms of Peri-implant diseases. J Dent Res. 2017:96(1):31-7.

10. Costa FO, Ferreira SD, Cortelli JR, Lima RPE, Cortelli SC, Cota LOM. Microbiological profile associated with peri-implant diseases in individuals with and without preventive maintenance therapy: a 5-year follow-up. Clin Oral Investig. 2019;23(8):3161-71.

11. Rakic M, Grusovin MG, Canullo L. The microbiologic profile associated with Peri-Implantitis in humans: a systematic review. Int J Oral Maxillofac Implants. 2016;31(2):359-68.

12. Lafaurie Gl, Sabogal MA, Castillo DM, Rincon MV, Gomez LA, Lesmes YA, Chambrone L. Microbiome and microbial biofilm profiles of Peri-Implantitis: a systematic review. J Periodontol. 2017;88(10):1066-89.

13. Colombo APV, Tanner ACR. The role of bacterial biofilms in dental caries and periodontal and Peri-implant diseases: a historical perspective. J Dent Res. 2019:98(4):373-85.

14. Mark Welch JL, Dewhirst FE, Borisy GG. Biogeography of the Oral microbiome: the site-specialist hypothesis. Annu Rev Microbiol. 2019;73: 335-58.

15. Al-Ahmad A, Muzafferiy F, Anderson AC, Wolber JP, Ratka-Kruger P, Fretwurst T, Nelson K, Vach K, Hellwig E. Shift of microbial composition of peri-implantitis-associated oral biofilm as revealed by $16 \mathrm{~S}$ rRNA gene cloning. J Med Microbiol. 2018;67(3):332-40.

16. Zhuang LF, Watt RM, Mattheos N, Si MS, Lai HC, Lang NP. Periodontal and peri-implant microbiota in patients with healthy and inflamed periodontal and peri-implant tissues. Clin Oral Implants Res. 2016;27(1):13-21.

17. Gao L, Xu T, Huang G, Jiang S, Gu Y, Chen F. Oral microbiomes: more and more importance in oral cavity and whole body. Protein Cell. 2018;9(5):488500.

18. Martellacci L, Quaranta G, Patini R, Isola G, Gallenzi P, Masucci L. A literature review of Metagenomics and Culturomics of the Peri-implant microbiome: current evidence and future perspectives. Materials. 2019;12(18):3010-9.

19. Reinedahl D, Chrcanovic B, Albrektsson T, Tengvall $P$, Wennerberg A. Ligature-Induced Experimental Peri-Implantitis-A Systematic Review. J Clin Med. 2018;7:12.

20. Sun J, Eberhard J, Glage S, Held N, Voigt H, Schwabe K, Winkel A, Stiesch M. Development of a peri-implantitis model in the rat. Clin Oral Implan Res. 2020;31(3):203-14

21. Zitzmann NU, Berglundh T. Definition and prevalence of peri-implant diseases. J Clin Periodontol. 2008;35(8 Suppl):286-91.

22. Monje A, Insua A, Wang HL. Understanding Peri-Implantitis as a PlaqueAssociated and Site-Specific Entity: On the Local Predisposing Factors. J Clin Med. 2019:8:2.

23. He CY, Jiang LP, Wang CY, Zhang Y. Inhibition of NF-kappa B by Pyrrolidine Dithiocarbamate prevents the inflammatory response in a ligature-induced Peri-Implantitis model: a canine study. Cell Physiol Biochem. 2018;49(2):61025

24. Huang BX, Zhang L, Xu L, Zhu WD, Witek L, Tovar N, Coelho PG, Meng HX. Effect of implant placement depth on the peri-implant bone defect configurations in ligature-induced peri-implantitis: an experimental study in dogs. Med Oral Patol Oral. 2018;23(1):E30-7.

25. Levin L, Zigdon H, Coelho PG, Suzuki M, Machtei EE. Reimplantation of dental implants following ligature-induced Peri-Implantitis: a pilot study in dogs. Clin Implant Dent R. 2013:15(1):1-6.

26. Lin XN, Liu T, Wu G, Zheng YN, Wismeijer D, Liu YL. Peri-implantitis induced by stainless steel ligature in beagle dogs. Int J Periodont Rest. 2017:37(3): E170-9.

27. Lawson PA, Johnson CN, Bengtsson L, Charalampakis G, Dahlen G, Moore E Falsen E. Peptostreptococcus canis sp nov., isolated from subgingival plaque from canine oral cavity. Anaerobe. 2012;18(6):597-601.

28. do Nascimento Silva A, de Avila ED, Nakano V, Avila-Campos MJ. Pathogenicity and genetic profile of oral Porphyromonas species from canine periodontitis. Arch Oral Biol. 2017:83:20-4.

29. Uitto VJ, Grenier D, Chan EC, McBride BC. Isolation of a chymotrypsinlike enzyme from Treponema denticola. Infect Immun. 1988:56(10):2717-22.

30. Tan KH, Seers CA, Dashper SG, Mitchell HL, Pyke JS, Meuric V, Slakeski N, Cleal SM, Chambers JL, McConville MJ, et al. Porphyromonas gingivalis and Treponema denticola exhibit metabolic symbioses. PLoS Pathog. 2014;10(3): e1003955.

31. Ma B, Wang Y, Ye S, Liu S, Stirling E, Gilbert JA, Faust K, Knight R, Jansson JK, Cardona $\mathrm{C}$, et al. Earth microbial co-occurrence network reveals interconnection pattern across microbiomes. Microbiome. 2020;8(1):82. 
32. Jiao S, Liu Z, Lin Y, Yang J, Chen W, Wei G. Bacterial communities in oil contaminated soils: biogeography and cooccurrence patterns. Soil Biol Biochem. 2016;98:64-73.

33. Apatzidou D, Lappin DF, Hamilton G, Papadopoulos CA, Konstantinidis A, Riggio MP. Microbiome of peri-implantitis affected and healthy dental sites in patients with a history of chronic periodontitis. Arch Oral Biol. 2017;83: $145-52$.

34. Sanz-Martin I, Doolittle-Hall J, Teles RP, Patel M, Belibasakis GN, Hämmerle $\mathrm{CHF}$, Jung RE, Teles FRF. Exploring the microbiome of healthy and diseased peri-implant sites using Illumina sequencing. J Clin Periodontol. 2017;44(12): 1274-84.

35. Kantarci A, Hasturk $H$, Van Dyke TE. Animal models for periodontal regeneration and peri-implant responses. Periodontology 2000. 2015;68(1): $66-82$.

36. Belibasakis GN, Mir-Mari J, Sahrmann P, Sanz-Martin I, Schmidlin PR, Jung RE. Clinical association of Spirochaetes and Synergistetes with peri-implantitis. Clin Oral Implants Res. 2016;27(6):656-61.

37. Escapa IF, Chen T, Huang Y, Gajare P, Dewhirst FE, Lemon KP. New Insights into Human Nostril Microbiome from the Expanded Human Oral Microbiome Database (eHOMD): a Resource for the Microbiome of the Human Aerodigestive Tract. mSystems. 2018;3:6.

38. Tzach-Nahman R, Mizraji G, Shapira L, Nussbaum G, Wilensky A. Oral infection with Porphyromonas gingivalis induces peri-implantitis in a murine model: evaluation of bone loss and the local inflammatory response. J Clin Periodontol. 2017;44(7):739-48.

39. Zhu B, Meng HX, Huang BX, Chen ZB, Lu RF. Detection of T-forsythia and other important bacteria in crestal and subcrestal implants with ligatureinduced peri-implant infection in dogs. J Periodontol. 2019;90(3):306-13.

40. Belkacemi S, Mazel A, Tardivo D, Tavitian P, Stephan G, Bianca G, Terrer E, Drancourt M, Aboudharam G. Peri-implantitis-associated methanogens: a preliminary report. Sci Rep. 2018;8(1):9447.

41. Faveri M, Figueiredo LC, Shibli JA, Perez-Chaparro PJ, Feres M. Microbiological diversity of peri-implantitis biofilms. Adv Exp Med Biol. 2015; 830:85-96.

42. Fickl S, Kebschull M, Calvo-Guirado JL, Hurzeler M, Zuhr O. Experimental Peri-Implantitis around different types of implants - a clinical and radiographic study in dogs. Clin Implant Dent Relat Res. 2015;17(Suppl 2): e661-9.

43. Liang J, Mai W, Tang J, Wei Y. Highly effective treatment of petrochemical wastewater by a super-sized industrial scale plant with expanded granular sludge bed bioreactor and aerobic activated sludge. Chem Eng J. 2019;360: $15-23$.

44. Edgar RC, Flyvbjerg H. Error filtering, pair assembly and error correction for next-generation sequencing reads. Bioinformatics. 2015;31(21):3476-82.

45. Edgar RC. UPARSE: highly accurate OTU sequences from microbial amplicon reads. Nat Methods. 2013;10(10):996-8.

46. Cole JR, Wang Q, Fish JA, Chai B, McGarrell DM, Sun Y, Brown CT, PorrasAlfaro A, Kuske CR, Tiedje JM. Ribosomal database project: data and tools for high throughput rRNA analysis. Nucleic Acids Res. 2014;42(Database issue):D633-42.

47. Wei Y, Ren T, Zhang L. Dix-seq: An integrated pipeline for fast amplicon data analysis. bioRxiv. 2020;2020:2005 2011.089748.

48. Watts SC, Ritchie SC, Inouye M, Holt KE. FastSpar: rapid and scalable correlation estimation for compositional data. Bioinformatics. 2019;35(6): 1064-6.

49. Otasek D, Morris JH, Boucas J, Pico AR, Demchak B. Cytoscape Automation: empowering workflow-based network analysis. Genome Biol. 2019;20:1.

\section{Publisher's Note}

Springer Nature remains neutral with regard to jurisdictional claims in published maps and institutional affiliations.

\section{Ready to submit your research? Choose BMC and benefit from:}

- fast, convenient online submission

- thorough peer review by experienced researchers in your field

- rapid publication on acceptance

- support for research data, including large and complex data types

- gold Open Access which fosters wider collaboration and increased citations

- maximum visibility for your research: over $100 \mathrm{M}$ website views per year

At $\mathrm{BMC}$, research is always in progress.

Learn more biomedcentral.com/submissions 